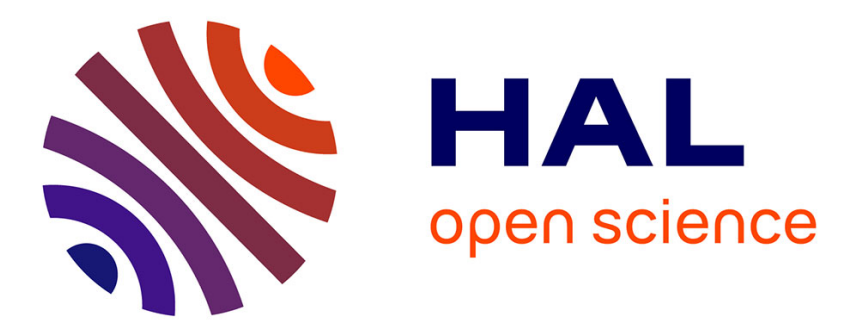

\title{
Hybrid 3D mass-spring system for simulation of isotropic materials with any Poisson's ratio
}

K. Golec, J.-F. Palierne, Florence Zara, S. Nicolle, Guillaume Damiand

\section{To cite this version:}

K. Golec, J.-F. Palierne, Florence Zara, S. Nicolle, Guillaume Damiand. Hybrid 3D mass-spring system for simulation of isotropic materials with any Poisson's ratio. The Visual Computer, 2020, 36 (4), pp.809-825. 10.1007/s00371-019-01663-0 . hal-02124471

\section{HAL Id: hal-02124471 https://hal.science/hal-02124471}

Submitted on 9 May 2019

HAL is a multi-disciplinary open access archive for the deposit and dissemination of scientific research documents, whether they are published or not. The documents may come from teaching and research institutions in France or abroad, or from public or private research centers.
L'archive ouverte pluridisciplinaire HAL, est destinée au dépôt et à la diffusion de documents scientifiques de niveau recherche, publiés ou non, émanant des établissements d'enseignement et de recherche français ou étrangers, des laboratoires publics ou privés. 


\title{
Hybrid 3D Mass-Spring System for simulation of isotropic materials with any Poisson's ratio
}

\author{
K. Golec · J.-F. Palierne · F. Zara - S. Nicolle · G. Damiand
}

Received: december 2017 / Accepted: april 2019

\begin{abstract}
Mass-Spring Systems (MSS) simulating elastic materials obey constraints known in elasticity as the Cauchy relations, restricting the Poisson ratio of isotropic systems to be exactly $v=1 / 4$. We remind that this limitation is intrinsic to centrosymmetric spring systems (where each node is a center of symmetry), forbidding them for instance to simulate incompressible materials (with $v=1 / 2$ ). To overcome this restriction, we propose to supplement the spring deformation energy with an energy depending on the volume only, insensitive to change of shape, permitting MSS to simulate any real isotropic materials. In addition, the freedom in choosing the spring constants realizing a given elastic behavior allows to manage instabilities. The proposed hybrid model is evaluated by comparing its response to various deformation geometries with analytical model and/or Finite Element Model (FEM). The results show that the hybrid MSS model allows to simulate any compressible isotropic elastic material and in particular the nearly incompressible (Poisson ratio $v \simeq 1 / 2$ ) biological soft tissues to which it is dedicated.
\end{abstract}

Keywords Physical Simulation - Mass-Spring System • Isotropic material

K. Golec · F. Zara · G. Damiand

Univ Lyon, Université Lyon 1, CNRS, LIRIS, UMR5205, F-69622, Lyon, France.

\section{J.-F. Palierne}

Univ Lyon, Université Lyon 1, CNRS, École Normale Supérieure de Lyon, Laboratoire de Physique, CNRS, UMR5672, Lyon, France.

S. Nicolle

Univ Lyon, Université Claude Bernard Lyon 1, IFSTTAR, LBMC UMR_T9406, F-69622 Lyon, France.

\section{Introduction}

From computer graphics to bioengineering applications, modeling soft materials subjected to large deformations is highly challenging. In particular, not only the realism of a deformation but also stability issues are still the main difficulties of the development of deformable object models, regardless of the domain of application. The needs and the requirements are however not the same, depending on whether the models are designed for animation or for scientific applications involving usually different computational approaches.

In computer graphics the computational speed and the visual realism of body deformation are main objectives in the development of new models. The mass-spring systems (MSS) meet these needs, as they are based on simple physical laws (relating the discretized acceleration of masses to the reacting spring forces) which allow fast computations and real-time topological changes (such as cutting or piercing). Therefore mass-spring systems are a great success among computer graphics developers [33, 14].

From another point of view, many new biomedical activities, such as the computer-based surgery, need soft material models which must represent biofidelic mechanical behavior while allowing real-time simulations. The main requirement of the biomedicine dedicated models is based on their robustness and the accuracy of their mechanical response (real 3D deformation and haptic forces). Since MSS can not properly simulate incompressible bodies, such as soft organs, because of the Cauchy constraints, existing instabilities and inability to derive a link between the physical body properties and the MSS parameters, scientists reckoned that the finite element method (FEM) was undeniably more relevant than the MSS for satisfying this need.

Using FEM gives the possibility of applying different physical laws in form of constitutive functions and precise boundary conditions to the model. Nevertheless, this better 
description of the mechanical behavior in case of FEM is counterbalanced by the advantages of mass-spring models, namely fast computation and effortless topological modifications in real-time without additional pre-computations (which are still necessary when using FEM). This holds for both linear and angular spring systems, as well as mixed systems [29].

To contribute to the effort of simulating soft tissues in real time, we propose a new formulation of the 3D MSS to improve its mechanical behavior for simulating any compressible isotropic soft materials while satisfying stability conditions. In particular, the MSS limitations prevent an arbitrary choice of the Poisson ratio (i.e. the ratio determining how a volume changes during a deformation). Thus in this paper we will focus on several solutions: (i) determining the stiffness constants of springs for 3D cubic symmetric MSS to reflect the Young modulus of the modeled body; (ii) addressing the stability issues of MSS; (iii) controlling the change of volume during a deformation process in agreement with the Poisson ratio of the modeled material.

In Section 2, we present the related work concerning simulation based on a mass-spring system. Section 3 presents the classical mass-spring model. Section 4 reminds the linear elasticity theory using a system based on springs. Some reminders about linear elasticity are given in Appendix A. Section 5 describes our approach to propose a hybrid massspring system (HybMSS) to deal with the limitations of the Cauchy theory. In this section, we address the formulation of our HybMSS (springs' configuration and their parameters) and the addition of correction forces to simulate materials with any Poisson's ratio. Section 6 presents the results of our experimentations followed by a discussion. Section 7 concludes this article and presents future work. Moreover, Table 1 gives the notations used in the article.

\section{Previous work}

Mass-spring models are widely used in computer graphics thanks to their fast computational capabilities [10]. They allow to simulate cloths in 2D [7] or deformable solids in $3 \mathrm{D}[35,34]$. They are based on an intuitive approach to simulate these elastic materials, with a discretization of an object into a set of particles (or masses) interconnected by a set of springs. However, the accuracy of their mechanical behavior is difficult to improve because of the local nature of deformations' treatment. It is also due to troubles to correctly adjust the stiffness of springs according to the mechanical properties of the materials [24,32] and difficulties to ensure the correct change of volume of compressible bodies. In this context, studies present attempting to reduce the impact of these issues over the years with several solutions.
Bourguignon [6] proposed an approach based on tetrahedral and hexahedral meshes to ensure the volume conservation of an element (with a maximal relative error $1.5 \%$ ). The volume-correcting force formulation uses information from springs (stiffness, damping) and vertices (positions, velocities) to tie the vertices to the barycenter of a volume instead of treating the vertex' forces only along the 3 main dimensions. However, since the hexahedral meshes are not homogeneous in their approach (the masses are not distributed evenly) the model can be used only for limited types of bodies. Also, the authors do not associate the volume-correcting forces to the actual volume of mesh elements.

Working on tetrahedral meshes, Mollemans [26] introduced an additional force term for the volume conservation. The force consists of 3 components: internal, external and corrective one (the latter factored by a constant, which is based on the influence of the elastic deformation and change of volume). The corrective force is based on the change of a volume of a basic element, but in the general calculations only the biggest forces are taken into account under condition $F>0.1 F_{\max }$. Such treatment ensures a good speed-up, but involves a major drawback for the accuracy.

Jarrousse [16] presented a simple method for volume conservation of myocardial non-linear anisotropic elastomechanical tissues. A hydrostatic work term (involving deformation tensor) is added to the energy density function, used later on to calculate volume preservation forces. However, such a method destabilizes the system, therefore the model is not suitable for real-time simulation.

Duan [12] presented novel compensations for 2D and 3D MSS, used in case of over-stretching or over-compressing a body. The concept called Position Based Mass-Spring System, has been inspired by Position Based Dynamics $[4,11]$. In this work springs are limited to $10 \%$ extension/compression to respect the natural behavior of materials. The results show mean error of $3.3 \%$ for small and $4.2 \%$ for non-linear deformations in comparison with FEM, proving model's usability for real organ simulation. Unfortunately, the method is not universal and the mesh-dependent deformation ratios need to be set up by the user to achieve best results.

To improve the accuracy, another approach involves incorporating the bio-mechanical properties of the simulated material into the formulation of the spring stiffness. Baudet [3] proposed a formulation based on the Young modulus and the Poisson ratio, and Natsupakpong [27] a formulation based on the Lamé constants. Moreover, Baudet [3] specifies forces to improve 2D and 3D MSS, but unfortunately they do not work with co-rotational modifications and they are based on an area-preserved energy. Moreover, the formulation of the Baudet forces was based on the assumption of small deformations (the Hooke law).

With the same approach, Kot [22,20,21] presented a method for overcoming the limitation of fixed Poisson's ratio when 
simulating elastic homogeneous isotropic bodies. His approach is verified on a cubic and disordered lattice. The method makes use of the existing vertices and corresponding distances between them, which makes the MSS look more like a meshless structure and behave like a fluid with no viscosity. Additional phenomenon which the authors introduce into the system is causing the nodes not only to repel each other, but also to radiate a dispersive momentum in random directions when two nodes start approaching each other. The approximated force of this momentum flow makes up for the desired value of Poisson's ratio. The formulation involves two adjustable parameters, which make the system behave differently at varied setup. The authors point out that when the Poisson ratio is changed, the corresponding change needs to be made to the bulk modulus to keep the Young modulus fixed. Unfortunately the method may increase the computation time twice.

Angular springs were considered by Kirkwood [19] in the study of molecular vibrations and later used in Sahimi and Arbabi's Bending Bond model [30,29]. Such springs, resisting changes of shape with no sensitivity to change of volume, allow to manipulate the shear modulus without changing the Bulk modulus, thereby varying the Poisson ratio.

Arnab and Raja [1] came up with an idea of additional force terms based on the change of volume and incorporating the bulk modulus into their 3D surface MSS model. This idea takes into account the elastic parameters of the simulated body (Young's modulus and Poisson's ratio), which the authors use to simulate breast surgery. The global deformation allows conservation of the volume, however the model itself is not generic and does not possess internal volume.

Born and Huang [5] give a comprehensive account of the dynamics and statics of crystals, using interatomic potentials as an essential ingredient instead of Cauchy's interatomic forces, resulting in a greater generality. Although the proper behavior of the general model under solid rotation has been questioned $[17,13]$, the Born-Huang theory of systems with central forces $[5, \S 11]$ is immune to such criticisms and, interestingly, applies to our mass-spring systems. Our treatment correspond indeed to Born and Huang's with the further simplification that, our springs being linear, the potentials are quadratic in the displacements.

Despite so many great improvements of MSS that are being made over the years, the problem of Cauchy's limitation of the fixed Poisson ratio (namely $v=1 / 4$ in 3D) persists for centrosymmetric spring systems. This article presents a new formulation of MSS supporting large deformations while preserving the isotropy of the material for any Poisson's ratio including negative. In this context, we propose a generic model, working regardless of the external forces and any topological modifications that the sample may undergo. The 3D structure is based on the formulation of the spring stiffness parameters according to the Young Modulus $E$ of the material, and the addition of correction forces based on the Poisson ratio $v$ of the material. The additional force term have been established from the volume strain energy to ensure a correct behavior of the simulated material during deformations.

\section{The classical mass-spring model}

The mass-spring model is based on the discretization of an object into a set of particles interconnected by springs. We denote $\mathbf{x}_{a}(t), \mathbf{v}_{a}(t)$ the position and velocity of particle $a$ at time $t, m_{a}$ being its mass. Moreover, the force acting on the particle $a$ exerted by the springs connected to it is given by

$\mathbf{f}_{a}^{(s)}(t)=\sum_{b} k_{a b} \mathbf{f}_{a b}^{(s)}=\sum_{b} k_{a b} \frac{\mathbf{x}_{b}(t)-\mathbf{x}_{a}(t)}{\left|\mathbf{x}_{b}(t)-\mathbf{x}_{a}(t)\right|}\left(\left|\mathbf{x}_{b}(t)-\mathbf{x}_{a}(t)\right|-l_{a b}\right)$

where $\mathbf{f}_{a b}^{(s)}(t)$ is the force exerted by the spring that connects the particle $a$ to the particle $b$, and $l_{a b}$ and $k_{a b}$ are the length at rest and the spring stiffness constant respectively. The length in the deformed state of the spring is then $l_{a b}^{\prime}(t)=$ $\left|\mathbf{x}_{b}(t)-\mathbf{x}_{a}(t)\right|$. Thus, the force exerted by the springs on a particle $a$ can be written

$\mathbf{f}_{a}^{(s)}(t)=\sum_{b} k_{a b}\left(\mathbf{x}_{b}(t)-\mathbf{x}_{a}(t)\right)\left(1-\frac{l_{a b}}{\left|\mathbf{x}_{b}(t)-\mathbf{x}_{a}(t)\right|}\right)$

To simulate the behavior of an object based on a MMS, the simulation loop is classically governed by the Newton's laws, thus deforming the initial lattice so that the MSS mimics the behavior of an elastic continuum. At each time $t$, the following computations are consequently performed on every particle of the MSS:

1. Computation of the total force $\mathbf{f}_{a}=\mathbf{f}_{a}^{(s)}+\mathbf{f}_{a}^{(g)}+\mathbf{f}_{a}^{(v)}$ acting on a particle $a$, namely the force $\mathbf{f}_{a}^{(s)}$ defined by equation (3.1) due to the springs connecting $a$ to neighboring particles and the gravitational force $\mathbf{f}_{a}^{(g)}=m_{a} \mathbf{g}$ with $\mathbf{g}$ being the gravitational acceleration. This force derives from the gravitational energy defined by $-\sum_{a} m_{a} \mathbf{g} \cdot \mathbf{x}_{a}$. To these classical forces, we add our correction force $\mathbf{f}_{a}^{(v)}$ which will be introduced in equation (5.15).

2. Computation of the acceleration of a particle $a$ at time $t$ according to Newton's second law:

$$
\frac{\partial \mathbf{v}_{a}(t)}{\partial t}=\frac{\mathbf{f}_{a}(t)}{m_{a}} . \quad \frac{\partial \mathbf{x}_{a}(t)}{\partial t}=\mathbf{v}_{a}(t)
$$

3. Computation of the new velocity $\mathbf{v}_{a}(t+h)$ and position $\mathbf{x}_{a}(t+h)$ of the particle $a$ using a numerical integration scheme like the Euler semi-implicit [28] or implicit one [2], with $h$ being the time step of the simulation. 


\begin{tabular}{|c|c|}
\hline Symbol & Explanation \\
\hline$t$ & time of a simulation \\
\hline$h$ & time step of a simulation \\
\hline $\mathbf{x}_{a}$ & position of a particle $a$ \\
\hline $\mathbf{v}_{a}$ & velocity of a particle $a$ \\
\hline$m_{a}$ & mass of a particle $a$ \\
\hline $\mathbf{f}_{a}$ & sum of the forces exerted on a particle $a$ \\
\hline $\mathbf{f}_{a}^{(g)}$ & force exerted by the gravity on a particle $a$ \\
\hline $\mathbf{f}_{a}^{(v)}$ & correction force exerted on a particle $a$ \\
\hline $\mathbf{f}_{a}^{(s)}$ & spring's force exerted on a particle $a$ \\
\hline $\mathbf{f}_{a b}^{(s)}$ & force exerted by a spring connecting particles $a, b$ \\
\hline$W$ & volume density of energy \\
\hline C & elasticity tensor \\
\hline$\varepsilon$ & strain tensor \\
\hline$\sigma$ & stress tensor \\
\hline$E$ & Young's modulus \\
\hline$v$ & Poisson's ratio \\
\hline$\lambda, \mu$ & Lamé coefficients \\
\hline K & shear modulus \\
\hline$G$ & bulk modulus \\
\hline$\kappa$ & additionnal coefficient of the bulk modulus \\
\hline$(s)$ & MSS \\
\hline$(s+i s o)$ & MSS with isotropy assumption \\
\hline (HybMSS) & hybrid MSS \\
\hline$($ HybMSS+iso $)$ & hybrid MSS with isotropy assumption \\
\hline $\begin{array}{l}V_{c} \\
V_{c}^{\prime}\end{array}$ & $\begin{array}{l}\text { volume of an undeformed cell } \\
\text { volume of a deformed cell }\end{array}$ \\
\hline $\begin{array}{l}r \\
r^{\prime} \\
\mathbf{a}, \mathbf{b}, \mathbf{c}\end{array}$ & $\begin{array}{l}\text { end-to-end vector of an undeformed spring } \\
\text { end-to-end vector of a deformed spring } \\
\text { edge vectors averaged over the edges of a cell }\end{array}$ \\
\hline $\begin{array}{l}k_{a b} \\
l_{a b} \\
l_{a b}^{\prime}\end{array}$ & $\begin{array}{l}\text { stiffness of a spring connecting particles } a, b \\
\text { length at rest of a spring connecting particles } a, b \\
\text { length of a spring connecting particles } a, b\end{array}$ \\
\hline $\begin{array}{l}l_{e}, k_{e} \\
l_{f}, k_{f} \\
l_{c}, k_{c}\end{array}$ & $\begin{array}{l}\text { characteristics of edge springs } \\
\text { characteristics of face diagonal springs } \\
\text { characteristics of cube diagonal springs }\end{array}$ \\
\hline
\end{tabular}

Table 1 Notations used in the article.

\section{Theoretical notions about springs system}

We want our springs system to reproduce the behavior of an elastic matter, especially of an isotropic matter. In annexe, we recall the theory of linear elasticity based on the macroscopic symmetry of the considered material, known as the multi-constant theory, as opposed to the rari-constant theory based on assumptions at the molecular level, to be considered thereafter. Note that a historical account of the rarivs multi-constant debate is given in [34].

4.1 The rari-constant theory: matter as a system of springs

Interested in the atomic constitution of matter, still hypothetical in his time, mathematician Augustin-Louis Cauchy studied the macroscopic state of stress of matter construed as an ensemble of molecules acting on one another through pairwise central forces, i.e. forces whose line of action passes through the interacting molecule's centers, assuming that the molecules are placed at centers of symmetry of the molecular arrangement $[9,8]$. In short, Cauchy modeled material bodies as mass-spring systems.
Following his lead, consider a spatially periodic lattice of cells of a volume $V_{c}$, each cell containing several springs labeled with index $\alpha$ (with no implicit summation over $\alpha$ ), of natural length $l_{\alpha}$ and spring constant $k_{\alpha}$. In a deformation that changes the spring length from $l_{\alpha}$ to $l_{\alpha}^{\prime}$, the volume density of spring elastic energy reads

$W^{(s)}=\frac{1}{2 V_{c}} \sum_{\alpha \in \text { cell }} k_{\alpha}\left(l_{\alpha}^{\prime}-l_{\alpha}\right)^{2}$.

Strictly speaking, $W^{(s)}$ is the energy per volume at rest, but the difference with the energy per deformed volume is of higher order and therefore immaterial in linear elasticity.

The dimension of the cell defines the microscopic scale and the deformation is assumed to vary over a much greater, macroscopic scale, the deformation gradient is thus nearly uniform at the scale of the cell. Consider therefore a homogeneous deformation $\mathbf{u}(\mathbf{x})=(\boldsymbol{\nabla} \mathbf{u})^{T} \cdot \mathbf{x}$ with uniform deformation gradient $\boldsymbol{\nabla} \mathbf{u}$ imposed on the system boundaries. Under Cauchy's assumptions of symmetry, the spring will deform affinely with the macroscopic deformation, their endto-end vector $\mathbf{r}_{\alpha}$ changing to $\mathbf{r}_{\alpha}^{\prime}=\mathbf{r}_{\alpha}+(\boldsymbol{\nabla u})^{T} \cdot \mathbf{r}_{\alpha}$ and their length changing from $l_{\alpha}=\sqrt{\mathbf{r}_{\alpha}^{2}}$ to

$l_{\alpha}^{\prime}=\sqrt{\mathbf{r}_{\alpha}^{\prime 2}}=l_{\alpha}+\frac{1}{l_{\alpha}} \nabla_{i} u_{j} r_{\alpha i} r_{\alpha j}=l_{\alpha}+\frac{1}{l_{\alpha}} \varepsilon_{i j} r_{\alpha i} r_{\alpha j}$

at first significant order in $\boldsymbol{\nabla u}$. Inserting this expression into the energy (4.1) yields

$$
\begin{aligned}
W^{(s)} & =\frac{1}{2 V_{c}} \sum_{\alpha \in \text { cell }} k_{\alpha}\left(\frac{1}{l_{\alpha}} \varepsilon_{i j} r_{\alpha i} r_{\alpha j}\right)^{2} \\
& =\frac{1}{2 V_{c}} \sum_{\alpha \in \text { cell }} \frac{k_{\alpha}}{l_{\alpha}^{2}} \varepsilon_{i j} \varepsilon_{k l} r_{\alpha i} r_{\alpha j} r_{\alpha k} r_{\alpha l}
\end{aligned}
$$

According to linear elasticity property (see appendix), the energy is also defined by $\frac{1}{2} C_{i j k l} \varepsilon_{i j} \varepsilon_{k l}$ which, for a springs system, involves:

$\mathrm{C}_{i j k l}^{(s)}=\frac{1}{V_{c}} \sum_{\alpha \in \mathrm{cell}} \frac{k_{\alpha}}{l_{\alpha}^{2}} r_{\alpha i} r_{\alpha j} r_{\alpha k} r_{\alpha l}$

The macroscopic elasticity tensor is thus completely symmetric with respect to its four (also interchanged) indices, thus supplementing symmetry relations with, what are now called, the Cauchy relations:

$\mathrm{C}_{i j k l}^{(s)}=\mathrm{C}_{i k j l}^{(s)}$,

which were pointed by Saint-Venant $[31, \S 12-13]$. These relations reduce the number of independent components of the stiffness tensor to 15 in the general case, hence the name rari-constant theory given to Cauchy's derivation of elasticity from microscopic arguments. 


\subsection{For isotropic materials}

In isotropic materials (see appendix), applying this relation to the isotropic stiffness tensor results in

$$
\lambda=G \quad \text { and } \quad C_{i j k l}^{(s+i s o)}=G\left(\delta_{i j} \delta_{k l}+\delta_{i k} \delta_{j l}+\delta_{i l} \delta_{j k}\right)
$$

where the shear modulus $G$ obtains by taking twice the trace of the stiffness tensor in (4.4) and (4.6) with:

$G=\frac{1}{15} C_{i i k k}^{(s+i s o)}=\frac{1}{15 V_{c}} \sum_{\alpha \in \text { cell }} k_{\alpha} l_{\alpha}^{2}$

The rari-isotropic material is thus described by one and only one elastic constant. The deformation energy then reduces to

$W^{(s+i s o)}=G\left(\frac{1}{2} \operatorname{Tr}(\varepsilon)^{2}+\operatorname{Tr}(\varepsilon)^{2}\right)=G\left(\frac{1}{2}\left(\varepsilon_{k k}\right)^{2}+\varepsilon_{i j} \varepsilon_{i j}\right)$.

Moreover, the rari-Young and Poisson moduli become

$E=\frac{5 G}{2}$

and

$v=1 / 4$

The Cauchy relation thus takes a particularly simple form: the Poisson modulus of isotropic spring systems cannot differ from $1 / 4$ [34].

\section{Our hybrid mass-spring system}

Real matter, however, happens to evade Cauchy's assumptions, thus multi-constant elasticity is in order for real materials, in contrast with mass-spring systems that provide perfect examples of rari-constant materials. Since our aim is to mimic real matter using numerical springs, this is an unfortunate limitation that leads us to supplement springs with a purely volume-dependent energy. By doing so, we allow $v$ to differ from $1 / 4$, as is the case in real matter. This route is opposite to that taken in the Bending-Bonds model, where linear springs are supplemented with angular springs sensitive to change of angles, thus reacting to change of shape but not to change of volume [30,29]. The Bending-Bonds model thus allows arbitrary Poisson ratios as well as ours but it seems at a greater computational cost.

\subsection{Our theoretical approach}

In our hybrid system (HybMSS), we consider the spring energy which is supplemented with a new term - quadratic in the relative volume change. We note $V_{c}^{\prime}$ the volume of the deformed cell. The volume density of the energy in a cell is thus written

$$
\begin{aligned}
W^{(H y b M S S)} & =W^{(s)}+W^{(v)} \\
& =\frac{1}{2 V_{c}} \sum_{\alpha \in \text { cell }} k_{\alpha}\left(l_{\alpha}^{\prime}-l_{\alpha}\right)^{2}+\frac{\kappa}{2}\left(\frac{V_{c}^{\prime}-V_{c}}{V_{c}}\right)^{2}
\end{aligned}
$$

where $\kappa$ is a coefficient relative to the material linked to the volume variation. Since the relative volume change is $\left(V_{c}^{\prime}-\right.$ $\left.V_{c}\right) / V_{c}=\varepsilon_{k k}$, we have (from 4.4)

$W^{(H y b M S S)}=\frac{1}{2} \mathrm{C}_{i j k l}^{(H y b M S S)} \varepsilon_{i j} \varepsilon_{k l}$,

with $\quad \mathrm{C}_{i j k l}^{(H y b M S S)}=\frac{1}{V_{c}} \sum_{\alpha \in \text { cell }} \frac{k_{\alpha}}{l_{\alpha}{ }^{2}} r_{\alpha i} r_{\alpha j} r_{\alpha k} r_{\alpha l}+\kappa \delta_{i j} \delta_{k l}$.

The new stiffness tensor $\mathbf{C}^{(H y b M S S)}$ being the sum of the spring stiffness tensor and a compression term proportional to $\kappa$.

For isotropic materials. If the spring system has been devised to be elastically isotropic (like that defined in next section), then one has

$W^{(\text {HybMSS+iso })}=\frac{1}{2}(G+\kappa)\left(\varepsilon_{k k}\right)^{2}+G \varepsilon_{i j} \varepsilon_{i j}$

where $G$ (being contributed by the springs) is still given by equation (4.7). The energy of the hybrid system for isotropic material therefore identifies with the multi-constant isotropic energy, with $\lambda=G+\kappa$.

If we consider an uniaxial strain experiment characterized by $\sigma_{x x} \neq 0, \sigma_{y y}=\sigma_{z z}=0$ which involves $\varepsilon_{y y}=\varepsilon_{z z}$, the Young and Poisson moduli are defined by $E=\sigma_{x x} / \varepsilon_{x x}$ and $v=-\varepsilon_{y y} / \varepsilon_{x x}$. Thus, the Young and Poisson moduli of the hybrid system are defined in terms of $G$ and $\kappa$ by:

$E=G \frac{3 \kappa+5 G}{\kappa+2 G} \quad$ and $\quad v=\frac{1}{2} \frac{\kappa+G}{\kappa+2 G}$

and the bulk modulus becomes

$K=\kappa+\frac{5}{3} G$.

Thus, $\kappa$ has a meaning of an additional bulk modulus.

Summary of our approach. The recipe for designing our theoretical hybrid system thus follows: (i) first make the size $l_{\alpha}$ small enough for the deformation to be approximately homogeneous over a cell, then (ii) adjust the spring constants to get the desired shear modulus $G$ (as presented in 
the next part as it depends on the geometry of the cell), and (iii) finally set $\kappa=K-\frac{5}{3} G, \kappa=G(4 v-1) /(1-2 v)$ or $\kappa=G(5 G-2 E) /(E-3 G)$ depending on whether $K$, $v$ or $E$ is prescribed. As a function of $E$ and $v$, one has $G=E /(2(1+v))$ and $\kappa=E(4 v-1) /((2(1+v)(1-2 v)))$.

The free setting of $\kappa$ and $\mu$ thus allows our HybridMSS to display any desired elastic constants, in contrast with the fixed $v=1 / 4$ that characterizes MSS (with $\kappa=0$ ). Note, however, that mechanical stability imposes $-1 \leq v \leq 1 / 2$ and that materials found in nature have $0 \leq v \leq 1 / 2$, the limits $v=0$ being reached by cork and $v=1 / 2$ corresponding to incompressible materials, i.e. materials with a shear modulus $\mu$ much smaller than the compression modulus $K$.

\subsection{Formulation of our HybMSS using cubical cells}

To verify our theoretical approach in practice, we pave the 3D space with a lattice of cubical MSS cells of edge length $l_{e}$ and volume $V_{c}=l_{e}{ }^{3}$ as shown in Fig. 1 .

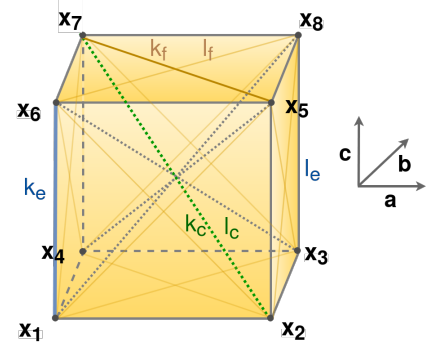

Fig. 1 An element of our MSS composed of 12 edge springs, 12 face diagonal and 4 inner diagonal springs interconnecting the particles.

A particle endowed with mass is thus affixed to each cube vertex, connected to neighboring particles by springs and acted upon by different forces. We chose to place not only springs on the edges, but also springs on the diagonals of the cells face and springs on the inner diagonals of the element. Thus, a cell of our HybMSS is composed of 12 springs (of stiffness constant $k_{e}$ and length $l_{e}$ ) spanning the edges, parallel to the axes directions, 4 springs (of stiffness constant $k_{c}$ and length $l_{c}=l_{e} \sqrt{3}$ ) spanning the cube diagonals placed inside the volume, and 12 springs (of stiffness constant $k_{f}$ and length $l_{f}=l_{e} \sqrt{2}$ ) spanning the face diagonals. In the undeformed state the springs belonging to any given cell have the following characteristics:

- Since any edge spring belongs to four adjacent cells, only one edge spring $\left(k_{e}, l_{e}\right)$ must be counted in each direction for a given cell. These springs span the following vectors of length $l_{e}$ :

$$
\mathbf{r}_{1}=\left(l_{e}, 0,0\right)^{T}, \quad \mathbf{r}_{2}=\left(0, l_{e}, 0\right)^{T}, \quad \mathbf{r}_{3}=\left(0,0, l_{e}\right)^{T} .
$$

- The four inner diagonal springs are represented by the following vectors of length $l_{c}=\sqrt{3} l_{e}$ :

$$
\begin{aligned}
& \mathbf{r}_{4}=\left(l_{e}, l_{e}, l_{e}\right)^{T}, \quad \mathbf{r}_{5}=\left(l_{e}, l_{e},-l_{e}\right)^{T}, \\
& \mathbf{r}_{6}=\left(l_{e},-l_{e}, l_{e}\right)^{T}, \quad \mathbf{r}_{7}=\left(-l_{e}, l_{e}, l_{e}\right)^{T} .
\end{aligned}
$$

- Since each face belongs to two adjacent cells, one must count once each of the following vectors of length $l_{f}=$ $\sqrt{2} l_{e}$

$$
\begin{aligned}
& \mathbf{r}_{8}=\left(l_{e}, l_{e}, 0\right)^{T}, \quad \mathbf{r}_{9}=\left(l_{e},-l_{e}, 0\right)^{T}, \mathbf{r}_{10}=\left(0, l_{e}, l_{e}\right)^{T} \\
& \mathbf{r}_{11}=\left(0, l_{e},-l_{e}\right)^{T}, \mathbf{r}_{12}=\left(l_{e}, 0, l_{e}\right)^{T}, \mathbf{r}_{13}=\left(l_{e}, 0,-l_{e}\right)^{T}
\end{aligned}
$$

Considering one element, the contribution of these springs to the stiffness tensor (4.4) is written, with $V_{c}=l_{e}^{3}$,

$$
\begin{aligned}
\mathrm{C}_{i j k l}^{(s)} & =l_{e}^{-3}\left\{\sum_{\alpha=1}^{3} l_{e}^{-2} k_{e} r_{\alpha i} r_{\alpha j} r_{\alpha k} r_{\alpha l}\right. \\
& +\sum_{\alpha=4}^{7} l_{c}^{-2} k_{c} r_{\alpha i} r_{\alpha j} r_{\alpha k} r_{\alpha l} \\
& \left.+\sum_{\alpha=8}^{13} l_{f}^{-2} k_{f} r_{\alpha i} r_{\alpha j} r_{\alpha k} r_{\alpha l}\right\} \\
& =l_{e}^{-5}\left\{k_{e} \sum_{\alpha=1}^{3} r_{\alpha i} r_{\alpha j} r_{\alpha k} r_{\alpha l}\right. \\
& \left.+\frac{k_{c}}{3} \sum_{\alpha=4}^{7} r_{\alpha i} r_{\alpha j} r_{\alpha k} r_{\alpha l}+\frac{k_{f}}{2} \sum_{\alpha=8}^{13} r_{\alpha i} r_{\alpha j} r_{\alpha k} r_{\alpha l}\right\} .
\end{aligned}
$$

By symmetry, components of the stiffness tensor $\mathbf{C}^{(s)}$ having an odd number of identical indices vanish and, directions 1, 2 and 3 being equivalent, the non-zero components of $\mathbf{C}^{(s)}$ are

$$
\begin{aligned}
\mathrm{C}_{1111}^{(s)} & =l_{e}^{-5}\left\{k_{e} \sum_{\alpha=1}^{3}\left(r_{\alpha 1}\right)^{4}+\frac{k_{c}}{3} \sum_{\alpha=4}^{7}\left(r_{\alpha 1}\right)^{4}+\frac{k_{f}}{2} \sum_{\alpha=8}^{13}\left(r_{\alpha 1}\right)^{4}\right\} \\
& =l_{e}^{-1}\left(k_{e}+\frac{4}{3} k_{c}+2 k_{f}\right) \\
& =\mathrm{C}_{2222}^{(s)}=\mathrm{C}_{3333}^{(s)}
\end{aligned}
$$

and

$$
\begin{aligned}
\mathrm{C}_{1122}^{(s)}= & l_{e}^{-5}\left\{k_{e} \sum_{\alpha=1}^{3}\left(r_{\alpha 1} r_{\alpha 2}\right)^{2}\right. \\
& \left.\quad+\frac{k_{c}}{3} \sum_{\alpha=4}^{7}\left(r_{\alpha 1} r_{\alpha 2}\right)^{2}+\frac{k_{f}}{2} \sum_{\alpha=8}^{13}\left(r_{\alpha 1} r_{\alpha 2}\right)^{2}\right\} \\
= & l_{e}^{-1}\left(\frac{4}{3} k_{c}+k_{f}\right) \\
= & \mathrm{C}_{1133}^{(s)}=\mathrm{C}_{2233}^{(s)}=\mathrm{C}_{1212}^{(s)}=\mathrm{C}_{1313}^{(s)}=\mathrm{C}_{2323}^{(s)},
\end{aligned}
$$

directly obtained from equation (5.6) 
The spring stiffness tensor thus has two independent components, as expected for a Cauchy-abiding system of cubic symmetry [34], (i.e invariant under $\pi / 2$ rotations about axes 1,2 and 3) whereas multi-constant theory allows three independent components for cubic systems [23].

Beyond its intrinsic cubic symmetry, our spring system can be tuned so as to be elastically isotropic. To that end, the constants $k_{e}, k_{c}$ and $k_{f}$ must be chosen in such a way that the spring stiffness tensor $\mathbf{C}^{(s)}$ can be cast into the form (4.6). This happens if and only if

$\mathrm{C}_{1111}^{(s)}=3 \mathrm{C}_{1122}^{(s)}$

leading to the elastic isotropy condition

$k_{e}=\frac{8}{3} k_{c}+k_{f}$,

and the shear modulus being

$G=\mathrm{C}_{1122}^{(s)}=l_{e}^{-1}\left(\frac{4}{3} k_{c}+k_{f}\right)$.

If $G$ is specified, we are left with one remaining degree or freedom. We choose it as a ratio $A=k_{f} / k_{c}$. Solving the system for the spring constants in terms of $A$ and $G$ or of $A$ and $E$ results in:

$$
\left\{\begin{array}{l}
k_{c}=\frac{3 G l_{e}}{4+3 A}=\frac{6}{5} \frac{E l_{e}}{4+3 A} \\
k_{f}=A k_{c} \\
k_{e}=G l_{e} \frac{8+3 A}{4+3 A}=\frac{2 E l_{e}}{5} \frac{8+3 A}{4+3 A}
\end{array}\right.
$$

The parameter $A$ ranges from 0 (vanishing face diagonal springs) to $\infty$ (vanishing cube diagonal springs).

\subsection{Formulation of our correction forces for cubical cells}

The volume $V_{c}^{\prime}$ of the deformed cell has no unambiguous definition since an arbitrary displacement of a cube's vertices does not necessarily send the four vertices of any face into a plane figure. We therefore introduce the following volume approximation

$V_{c}^{\prime}=\mathbf{a} \cdot(\mathbf{b} \times \mathbf{c})$,

where $\mathbf{a}, \mathbf{b}, \mathbf{c}$ are edge vectors averaged over edges of the cell (Fig 1) defined by:

$$
\left\{\begin{array}{l}
\mathbf{a}=\frac{1}{4}\left(\mathbf{x}_{2}-\mathbf{x}_{1}+\mathbf{x}_{3}-\mathbf{x}_{4}+\mathbf{x}_{5}-\mathbf{x}_{6}+\mathbf{x}_{8}-\mathbf{x}_{7}\right) \\
\mathbf{b}=\frac{1}{4}\left(\mathbf{x}_{3}-\mathbf{x}_{2}+\mathbf{x}_{4}-\mathbf{x}_{1}+\mathbf{x}_{8}-\mathbf{x}_{5}+\mathbf{x}_{7}-\mathbf{x}_{6}\right) \\
\mathbf{c}=\frac{1}{4}\left(\mathbf{x}_{6}-\mathbf{x}_{1}+\mathbf{x}_{8}-\mathbf{x}_{3}+\mathbf{x}_{5}-\mathbf{x}_{2}+\mathbf{x}_{7}-\mathbf{x}_{4}\right)
\end{array}\right.
$$

This formula gives the exact volume for homogeneous deformations of the original cell, however strong this homogeneous deformation is, and provides an approximation in inhomogeneous deformations (inhomogeneously deformed cells, however, call for mesh refinement). As required on physical grounds [18], expression (5.1-5.13) of the compression energy $W^{(v)}$ is invariant in solid rotations, leaving $V_{c}^{\prime}$ and $W^{(v)}$ unchanged. Expression (5.13) however infringes the proscription of pseudoscalar products in the Hamiltonians of physical systems [18, note 5], with the consequence that $W^{(v)}$ is not invariant in central inversions, which change $V_{c}^{\prime}$ into $-V_{c}^{\prime}$. Such transformations, however, must pass through the singular point $V_{c}^{\prime}=0$, an event that will not happen in linear elasticity. Moreover, our system thus avoids locking into inverted configurations, their energy being unfavourable. Note that changing $\left(\left(V_{c}^{\prime}-V_{c}\right) / V_{c}\right)^{2}$ for $\left(\left(V_{c}^{\prime 2}-V_{c}^{2}\right) /\left(2 V_{c}^{2}\right)\right)^{2}=$ $\left(\left(V_{c}^{\prime}-V_{c}\right) / V_{c}\right)^{2}+\mathscr{O}\left(V_{c}^{\prime}-V_{c}\right)^{3}$ in equation (5.1) would result in the same linear elastic behavior whilst avoiding the pseudoscalar product, since $V_{c}^{\prime 2}$ can be expressed as a combination of scalar products of the vectors $\mathbf{a}, \mathbf{b}$ and $\mathbf{c}$, to the expense of both an increased computational complexity and a recovered propensity to lock into inverted configurations.

The correction force on a particle $a$ derives from the volume-dependent term of the energy density $W^{(\text {HybMSS })}$ with:

$$
\begin{aligned}
\mathbf{f}_{a}^{(v)} & =-\frac{\partial W^{(v)}}{\partial \mathbf{x}_{a}}=-\frac{\partial}{\partial \mathbf{x}_{a}}\left(\frac{\kappa}{2} \frac{\left(V_{c}^{\prime}-V_{c}\right)^{2}}{V_{c}}\right) \\
& =-\kappa \frac{V_{c}^{\prime}-V_{c}}{V_{c}} \frac{\partial V_{c}^{\prime}}{\partial \mathbf{x}_{a}} \\
& =-\kappa\left(l_{e}^{-3} \mathbf{a} \cdot(\mathbf{b} \times \mathbf{c})-1\right) \frac{\partial V_{c}^{\prime}}{\partial \mathbf{x}_{a}}
\end{aligned}
$$

The gradient of $V_{c}^{\prime}$ with respect to the positions of particles 1 to 8 is easily found to be

$\frac{\partial V_{c}^{\prime}}{\partial \mathbf{x}_{1}}=-\frac{\partial V_{c}^{\prime}}{\partial \mathbf{x}_{8}}=-(\mathbf{b} \times \mathbf{c})-(\mathbf{c} \times \mathbf{a})-(\mathbf{a} \times \mathbf{b})$,

$\frac{\partial V_{c}^{\prime}}{\partial \mathbf{x}_{2}}=-\frac{\partial V_{c}^{\prime}}{\partial \mathbf{x}_{7}}=(\mathbf{b} \times \mathbf{c})-(\mathbf{c} \times \mathbf{a})-(\mathbf{a} \times \mathbf{b})$,

$\frac{\partial V_{c}^{\prime}}{\partial \mathbf{x}_{3}}=-\frac{\partial V_{c}^{\prime}}{\partial \mathbf{x}_{6}}=(\mathbf{b} \times \mathbf{c})+(\mathbf{c} \times \mathbf{a})-(\mathbf{a} \times \mathbf{b})$,

$\frac{\partial V_{c}^{\prime}}{\partial \mathbf{x}_{4}}=-\frac{\partial V_{c}^{\prime}}{\partial \mathbf{x}_{5}}=-(\mathbf{b} \times \mathbf{c})+(\mathbf{c} \times \mathbf{a})-(\mathbf{a} \times \mathbf{b})$.

It must be kept in mind that, since any particle belongs to eight neighboring cells, the total correction force on this particle is the sum of the contributions of the considered cells. The computational cost of equation (5.16) compares favorably with that of forces derived from angular springs: see [30, eq.3] and terms in $\beta$ in [29, eq.2.27-28]. 


\subsection{Assembly of cubical hybrid MSS cells}

To simulate the mechanical behavior of a complex object, we represent it by an assembly of our 3D hybrid MSS cells. To create the whole hybrid MSS, we proceed as following:

- The global mass of the object is distributed on the different particles of the system. That is, considering a 3D object of mass density $\rho$, the mass $m_{a}$ of each particle $a$ is defined by $m_{a}=\sum_{j} \frac{\rho}{8} V_{c}^{j}$ with $\sum_{j}$ being the sum over all the cubical elements to which $m_{a}$ belongs, with volumes defined by $V_{c}^{j}$.

- An edge spring has a stiffness $k_{e} / 4$ if the edge is not shared between several elements. Then, the Kirchhoff's law is applied: for example an edge spring has a stiffness $k_{e}$ if the edge is in the middle of the lattice (i.e. with 4 neihboring cubical elements).

- A face diagonal spring has a stiffness $k_{f} / 2$ if the considered face is not common with any other element (i.e. for the faces at the boundaries of the mesh). Then, the Kirchhoff's law is applied: for example the face diagonal springs have a stiffness $k_{f}$ if the considered face is common with 2 elements.

\section{Experimental results}

We compared our mass-spring system using correction forces with and witout face diagonal springs, with experiments performed using LS-Dyna software [15], which is a tool performing finite element (FEM) simulations. We used LS-Dyna version 971dR4 (LSCT, Livermore, CA, USA) with the elastic law and implicit solution for the material simulated.

\subsection{The simulated experimentations}

A deformation of a material can be decomposed into 5 basic experiments (shown in Fig. 2): shearing, tension, compression, bending and torsion. Our objective is to study the response of the body to all the types of tests. Each of the main experiments was performed with the same set of parameters and using three simulation method cases:

- Our hybrid mass-spring system with only edge and innerdiagonal springs (i.e. without face diagonal springs) (denoted HybMSS-FD), using the formulation of stiffness defined in (5.12); the parameter $A$ is set to 0 to simulate no face-diagonal springs; and to be able to use full range of the Poisson ratio we apply the correction forces;

- Our hybrid mass-spring system with face diagonal springs and correction forces (i.e. our full formulation of part (5.2)) (denoted HybMSS);

- Finite Element Method (denoted FEM).
In all the instances except of the beam bending experiment (which is examined at equilibrium), we fixed the timestep to $0.001 \mathrm{~s}$, and the total number of iteration steps to 10,000 (i.e. 10 s). No gravity is used in those experiments. For the correction forces expressed in (5.15), we used the compression modulus $\kappa$ with $v \neq 1 / 2$. Note, that in case of $v=1 / 4$ the correction forces are equal to 0 , as the massspring system naturally models this case.

The tensile and the beam bending experiments were performed on a beam of a size $0.4 \times 0.4 \times 2.4 \mathrm{~m}^{3}$. Two different mesh densities were used for both experiments: $4 \times 4 \times 24$ and $8 \times 8 \times 48$ elements. Additionally, for the beam flexion we use the gravity constant $g=-10 \mathrm{~m} / \mathrm{s}^{2}$.

All the other experiments were performed on a sample of $10 \times 10 \times 10$ cubes of a unit size $0.5 \mathrm{~m}$, which makes a sample of total volume $V_{0}=125 \mathrm{~m}^{3}$. The corresponding FEM experiments were performed on samples of size matching exactly the ones used for mass-spring system tests.

\subsection{Comparison between the several approaches}

The deformations were limited to $5 \%$ and $20 \%$. The detailed parameters description and results for the compression and tension experiments after 10 seconds of deformation process are presented in Tables 2 and 3. The column $e$ shows the relative change of the volume computed using the following formula: $e=\left|V_{c}-V_{c}^{\prime}\right| / V_{c}$ to validate body's volume behavior during simulations. Below we present the comparison of different approaches (HybMSS-FD, HybMSS, FEM and/or analytical solution) for the previously introduced test cases.

Compression. In Fig. 3, showing the compression experiments, we can see a great difference in the behavior of the samples. The wrinkle instability is very noticeable in experiments on HybMSS-FD (a)-(c), which is clearly 'wrinkled' due to the lack of springs in the face. In the same time, both HybMSS (e-f) and FEM (h-i) show slightly 'swollen' but regular figure with divergent volume distribution. Incompressible part of this experiment shows slightly different image though. Unlike all the other experimental cases, the FEM compression (i) is also slightly 'wrinkled', which was definitely not an expected outcome. Here the impact of the correction forces is visible the best, paired with the visually correct solution and smooth walls of the HybMSS sample. Additionally we can verify the numerical results of HybMSS from Table 2 with the compressible $(v=0)$ HybMSS experiment in the figure and see that the walls of the cube are perfectly straight and the compressibility rate matches the theoretical one, exactly as expected.

Tension. Fig. 4 showing tensile test results is the opposite of the compression experiment. Here, taking into account the numerical data, we can see that the volume of the wholly compressible HybMSS (d) has a very regular shape, with the horizontal walls staying parallel to the $X$ axis, without 


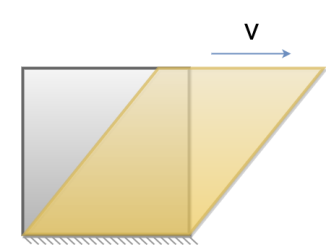

(a) Shearing

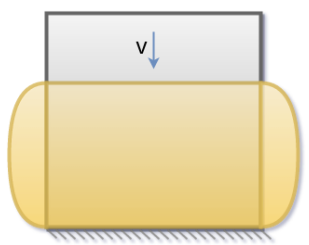

(b) Compression

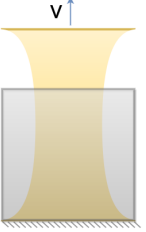

(c) Tension

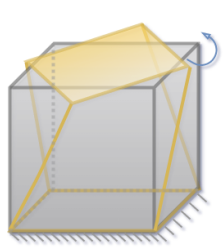

(d) Torsion

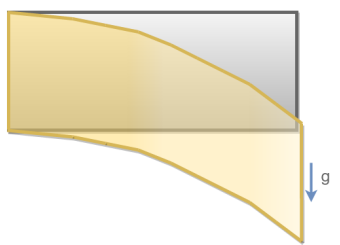

(e) Bending

Fig. 2 Illustrations of the five test cases performed to validate our hybrid 3D mass-spring system.

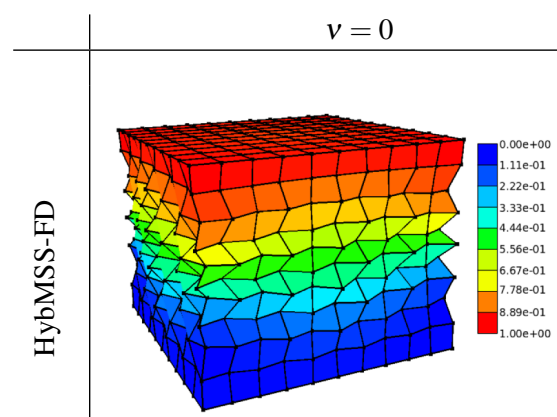

(a)

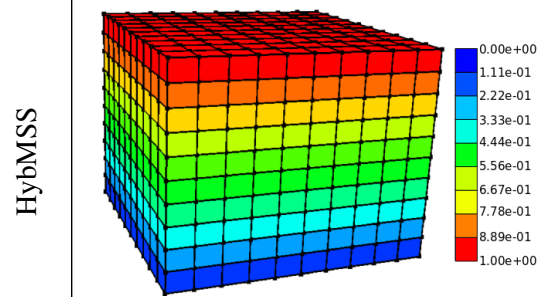

(d)

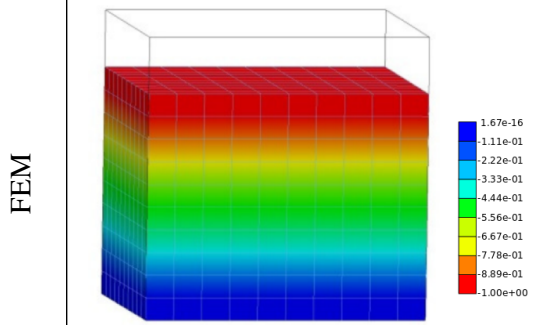

(g)

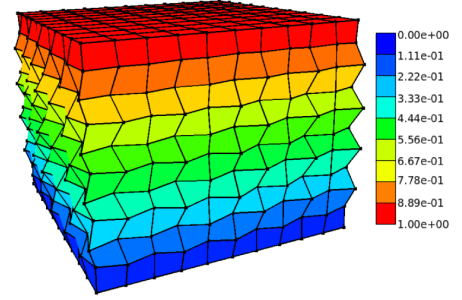

(b)

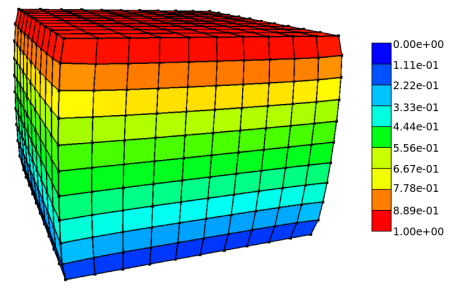

(e)

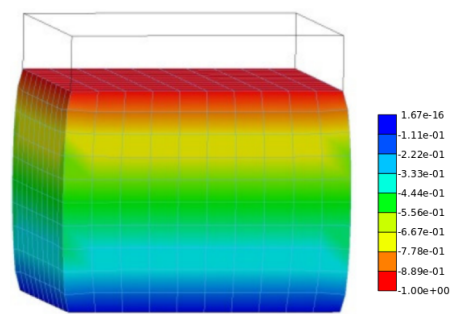

(h)

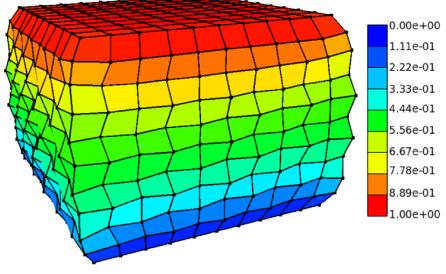

(c)

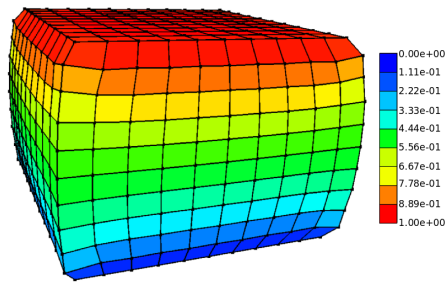

(f)

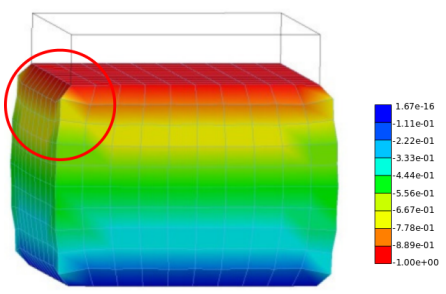

(i)

Fig. 3 Compression experiment comparison between HybMSS-FD, HybMSS and FEM at a big (20 \%) deformation with different Poisson's ratios. The color scale corresponds to the displacement from the original position.

any curve, similarly to the FEM solution with the same parameters (g) and the HybMSS-FD experiment (a). We owe this shape to the correction forces, which 'guard' the correct volume changes within the samples. We can also observe that the distribution of the volumes is much improved between HybMSS-FD (b)-(c) and HybMSS (e)-(f). In contrast to that, all HybMSS-FD (b-c), HybMSS (e, f) and FEM (h, i) show a nice flex curve, which is slightly too deep for the HybMSS-FD simulation at $v=1 / 4$. Additionally, according to Table 3, even the correction forces cannot change the huge impact, which is made by the lack of face-diagonal springs, making the variations between HybMSS-FD and HybMSS volumes noticeable.

In every experiment presented in Tables 2 and 3, the differences between all the models are observable. However, the highest relative error's value of the volume conserving HybMSS-FD models $(v=0.499)$ reaches $0.01 \%$, even without the use of face-diagonal springs. The biggest deviation of the HybMSS from the FEM appeared in the $20 \%$ deformation in tensile test with $v=0.25$ and it differs by $1.21 \%$, 


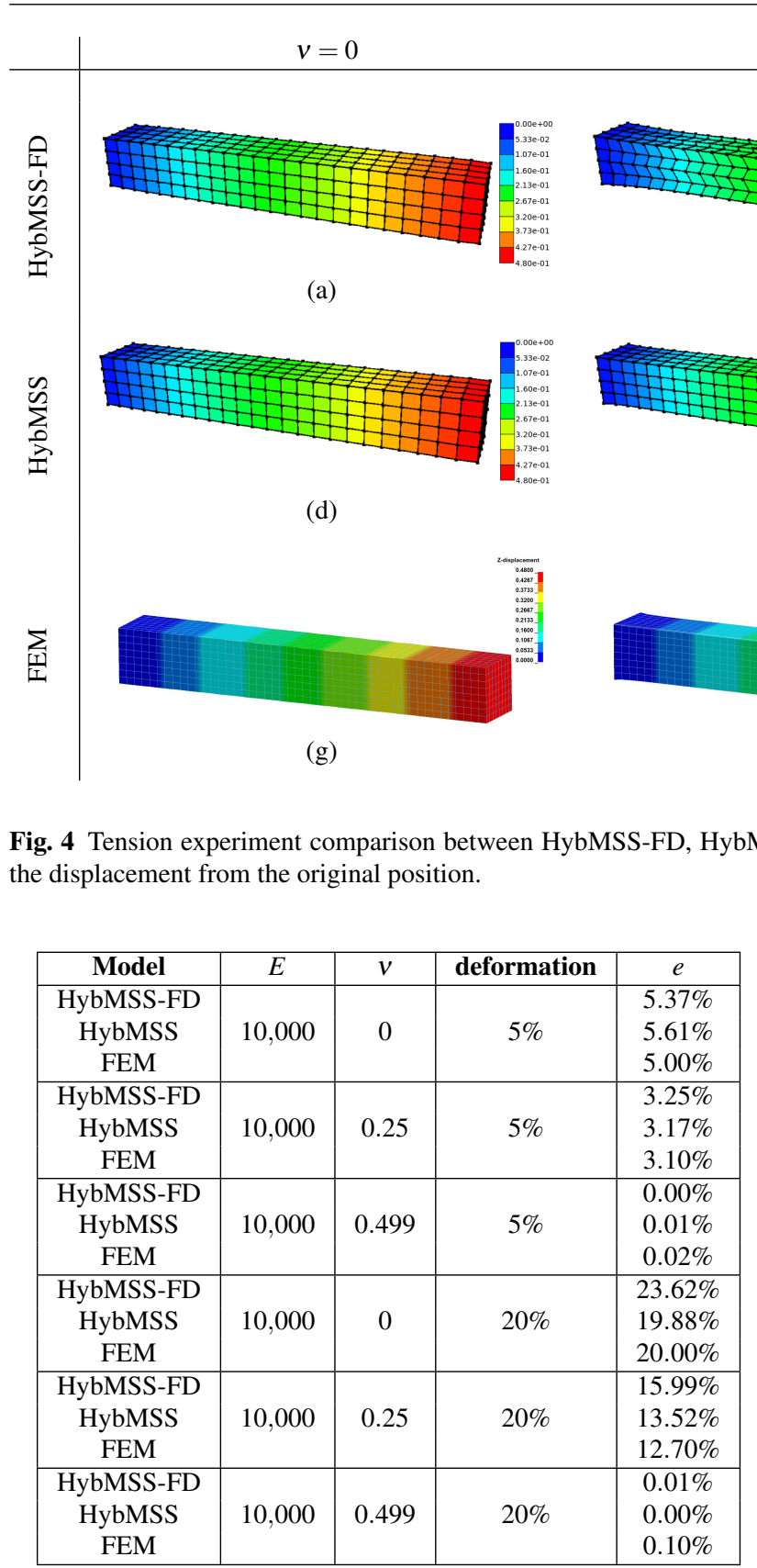

Table 2 Testing parameters and results of the compression.

which is a very acceptable difference to say the least. We can easily see that the change of the HybMSS volume in comparison to FEM has a very small deviation. However it is essential to compare these numerical results with the actual shape deformations, as we did, to see the real role of the correction forces matched with the use of full set of springs.

Beam bending. Fig. 5 presents the results of the beam under gravity experiment in comparison with the analytical curve computed using the following Euler-Bernoulli equation [25]:

$y(x)=\frac{\rho g l h}{24 E I}\left(4 L x^{3}-6 L^{2} x^{2}-x^{4}\right)$. $v=0.25$

$v=0.499$

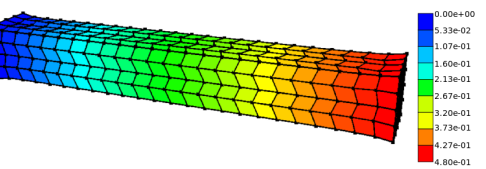

(c) (b)

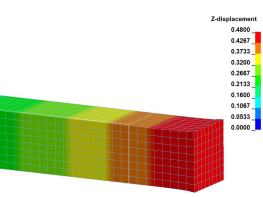

(h)

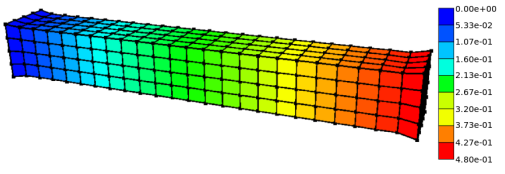

(f)

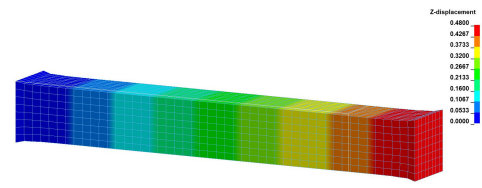

(i)

\begin{tabular}{|c|c|c|c|c|}
\hline Model & $E$ & $v$ & deformation & $e$ \\
\hline HybMSS-FD & & & & $7.46 \%$ \\
HybMSS & 10,000 & 0 & $5 \%$ & $4.40 \%$ \\
FEM & & & & $5.00 \%$ \\
\hline HybMSS-FD & & & & $0.85 \%$ \\
HybMSS & 10,000 & 0.25 & $5 \%$ & $2.54 \%$ \\
FEM & & & & $2.61 \%$ \\
\hline $\begin{array}{c}\text { HybMSS-FD } \\
\text { HybMSS }\end{array}$ & 10,000 & 0.499 & $5 \%$ & $0.00 \%$ \\
FEM & & & & $0.00 \%$ \\
HybMSS-FD & & & & $0.00 \%$ \\
HybMSS & 10,000 & 0 & $20 \%$ & $2.92 \%$ \\
FEM & & & & $19.99 \%$ \\
\hline HybMSS-FD & & & & $3.15 \%$ \\
HybMSS & 10,000 & 0.25 & $20 \%$ & $8.90 \%$ \\
FEM & & & & $10.11 \%$ \\
\hline HybMSS-FD & & & & $0.00 \%$ \\
HybMSS & 10,000 & 0.499 & $20 \%$ & $0.00 \%$ \\
FEM & & & & $0.00 \%$ \\
\hline
\end{tabular}

Table 3 Testing parameters and results of the tension simulations.

It takes into account the parameters: $l, h$ - width and height of a beam, $L$ - the length of the beam, $\rho$ - the body density, gravity $g$ and the moment of inertia $I=l h^{3} / 12$. It is visible with two beam densities that the curve seems to converge to the analytical solution.

On the other hand we can compare also the results of HybMSS with HybMSS-FD on the same figure. HybMSSFD beam is much softer and even though the coarser beam is close to the analytical solution, we can see that with the change of mesh resolution the beam becomes softer than the correct result. 
An interesting property of the beam can be observed on Fig. 6, where in the HybMSS-FD case there are apparent wrinkles at the compressed part of the beam, which are absent when face diagonal springs are used. We can see the impact of those springs very clearly.
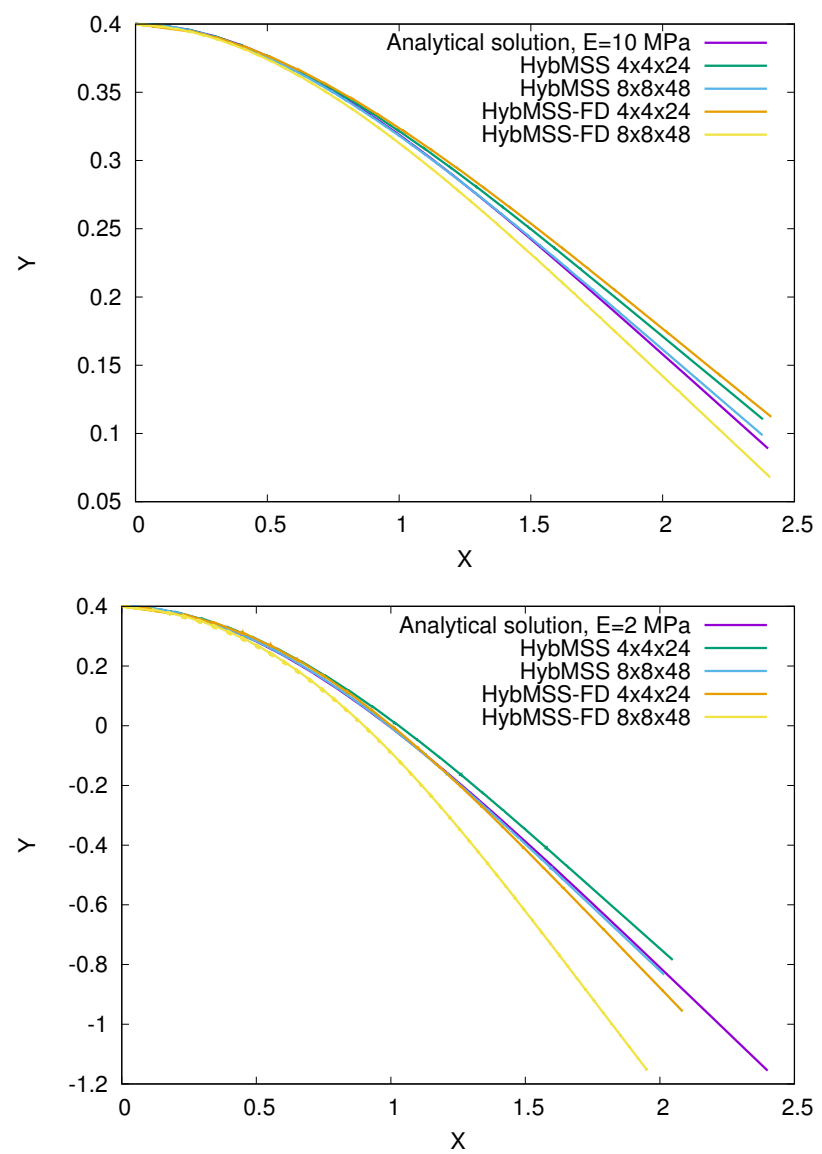

Fig. 5 Beam under gravity comparison between HybMSS, HybMSS$\mathrm{FD}$ and the analytical solution. $E=10 \mathrm{MPa}$ (upper), $E=2 \mathrm{MPa}$ (lower), $v=0.25, g=-10, \mathbf{A}=1$.
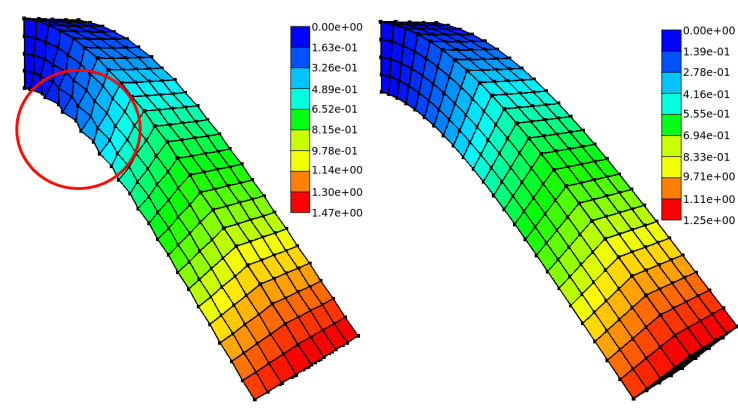

Fig. 6 Beam under gravity: HybMSS-FD (left), HybMSS (right). $E=$ $2 \mathrm{MPa}, v=0.25, g=-10$. The color scale corresponds to the displacement from the original position.
Shearing. In case of the shearing experiment (see Fig. 7) we can study the shape of the body on Fig. 8. The plots represent the cross-sectional view of the middle layer (meaning $Z=5$ ) in the $X-Y$ plane. A slight wave on the layers of the sample, which is in fact a natural behavior of an elastic body, is an obligatory element of this type of deformation. Here, the correction forces ensure smoothness of the sample at every layer, and the difference between the HybMSS and FEM is minor, since it is the role of the inner diagonal springs to deform the volumes correctly at this type of deformation.

Additionally, Table 4 presents a study of the order of magnitude of the top plate response to deformation for shearing experiment with Poisson's ratio $v=1 / 4$. The presented values represent force $([\mathrm{N}])$ and we can see that for HybMSSFD and HybMSS they do not differ much from the FEM, which is exactly our objective.

\begin{tabular}{|c|c|c|c|}
\hline Deformation & HybMSS-FD & HybMSS & FEM \\
\hline $5 \%$ & 3,390 & 3,396 & 3,317 \\
$20 \%$ & 14,662 & 14,600 & 13,234 \\
\hline
\end{tabular}

Table 4 Response of the top plate in shearing deformation with $E=$ $10,000, v=0.25$. The measured response is force $[\mathrm{N}]$.

We can also study the average difference of the HybMSS and FEM positions at this layer, which for the Poisson ratio values $v=0, v=0.25$ and $v=0.499$ are respectively: $0.042 \mathrm{~m}, 0.013 \mathrm{~m}$ and $0.019 \mathrm{~m}$. Let us remind that the samples are of a wall length $5 \mathrm{~m}$.

Torsion. Adequately, Fig. 9 shows the results of torsion experiment. We compare the cross-sectional view of the middle layer $(Y=5)$ on $X-Z$ plane of a deformed body in Fig. 10. The experiments are visibly smooth and we can see how close the HybMSS is to the FEM solution. Here again we can verify the average difference of the HybMSS and FEM point's positions to additionally confirm the HybMSS model's accuracy. For the Poisson ratio values $v=0, v=$ 0.25 and $v=0.499$ the average difference between points reaches respectively: $0.064 \mathrm{~m}, 0.030 \mathrm{~m}$ and $0.008 \mathrm{~m}$.

Similarly as in case of the shearing experiment, Table 5 presents a study of the order of magnitude of the top plate response to torsional deformation, also with Poisson's ratio $v=1 / 4$. We can see that the order of magnitude of the HybMSS-FD and HybMSS experiments correspond with the FEM solution, which we owe to the correction forces and physically based stiffness formulation.

\begin{tabular}{|c|c|c|c|}
\hline Deformation & HybMSS-FD & HybMSS & FEM \\
\hline $5 \%$ & 1,277 & 1,306 & 1,279 \\
$20 \%$ & 60,005 & 60,565 & 57,981 \\
\hline
\end{tabular}

Table 5 Response of the top plate in torsion deformation with $E=$ $10,000, v=0.25$. The measured response is torque $[\mathrm{N} \cdot \mathrm{m}]$. 


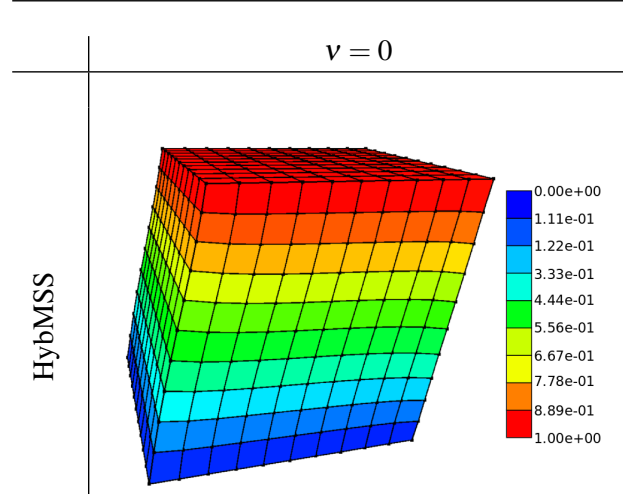

(a)

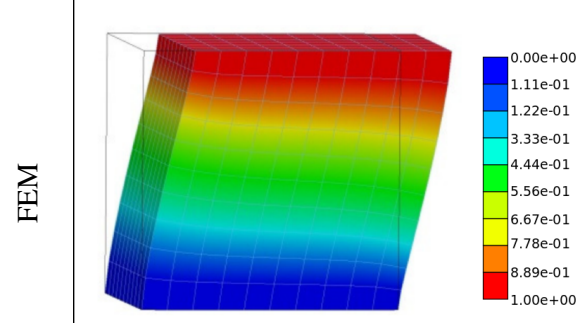

(d) $v=0.25$

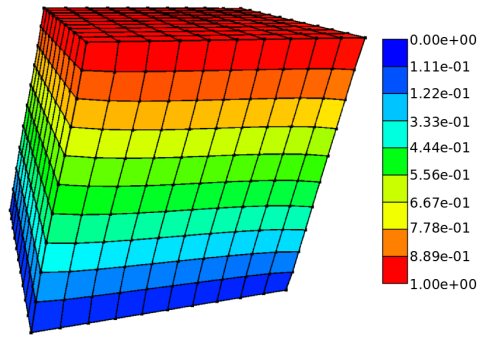

(b)

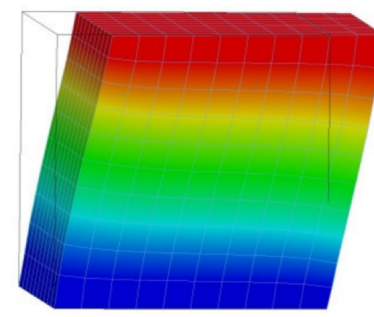

(e) $v=0.499$

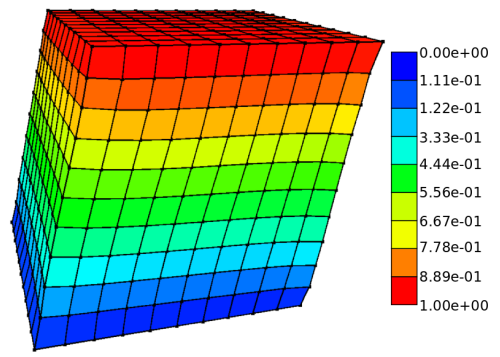

(c)

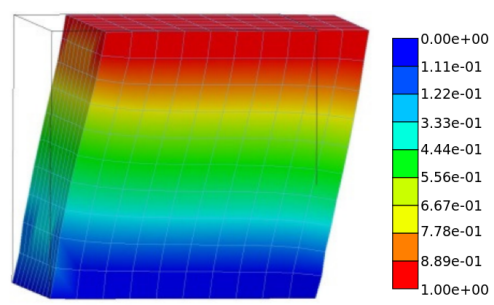

(f)

Fig. 7 Shearing experiment with big (20\%) deformation at different Poisson's ratios, $E=10,000$; comparison between HybMSS and FEM. The color scale corresponds to the displacement from the original position.

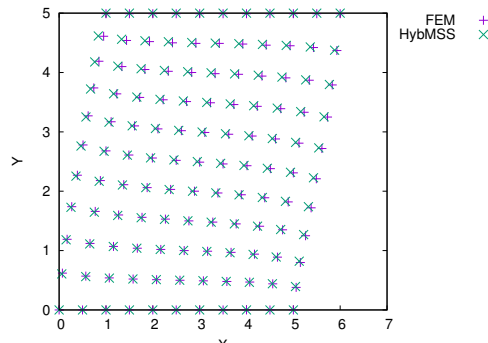

(a) $v=0$

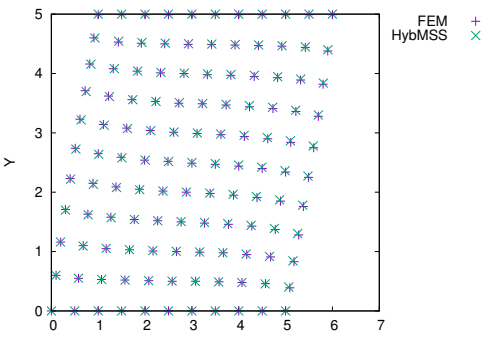

(b) $v=0.25$

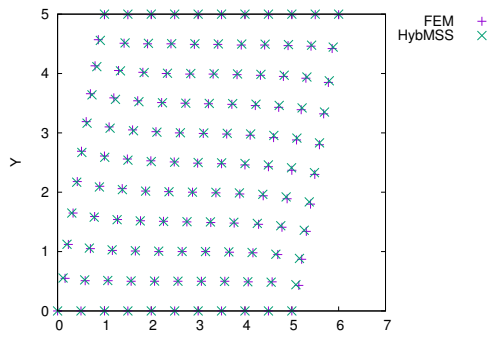

(c) $v=0.499$

Fig. 8 Shape of the body for the shearing experiment with big (20\%) deformation at different Poisson's ratios, $E=10,000$; comparison between HybMSS and FEM.

\subsection{Performance Study}

We compared the performance of our two approaches, HybMSS FD and HybMSS, with a third version called HybMSS-CF which has face diagonal springs but without correction forces. This comparison allows us to evaluate the impact of the addition of the face diagonal springs and the impact of the computation of the correction forces. These tests were achieved on a Intel ${ }^{\circledR}$ Core $^{\mathrm{TM}}$ i7 - 4790K @ 4.00 GHz CPU.

Two experiments were performed, compression and beam under gravity, with 4 different mesh sizes for both cases. The first four meshes are cubical and the last four are beams of a $1: 1: 6$ length ratio. The cause for using two types of meshes (cube and beam) is to show how the performance changes depending on the type of deformation. As we can see in Table 6, the number of springs for HybMSS-FD is much smaller that for HybMSS and for HybMSS-CF (about

\section{5 times bigger).}

We decided to execute 1,000 iterations of the compression experiment; 300,000 iterations of the beam flexion test. Fig. 11 and 12 show the two experiments (compression and beam under gravity) performance plotted versus the number of mesh volumes. Of course the expected outcome of these simulations was that HybMSS-CF method will have slightly higher execution time than the simpler HybMSS-FD, due to the difference of the number of springs. Then, HybMSS performed even worse, since the number of springs was already increased and it is necessary to compute the force corrections for each volume separately. 


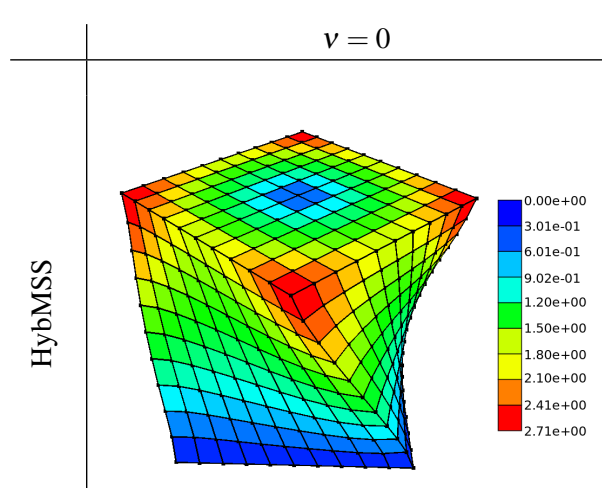

(a)

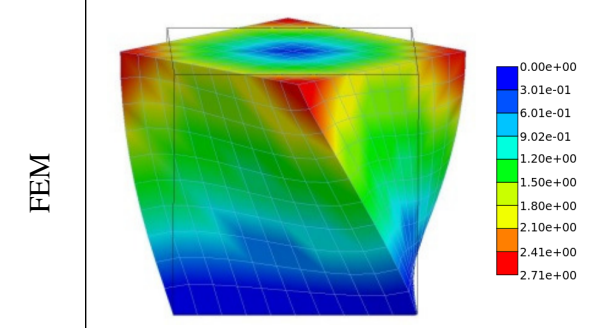

(d)

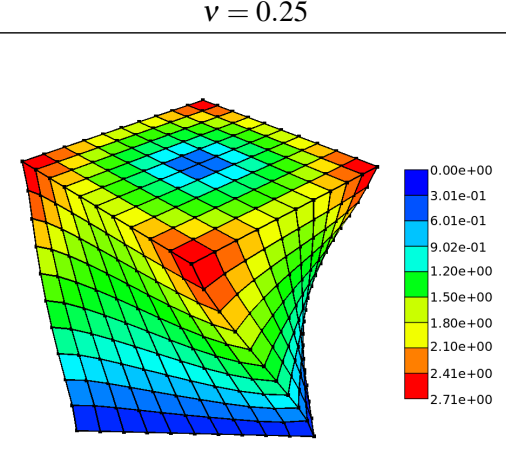

(b)

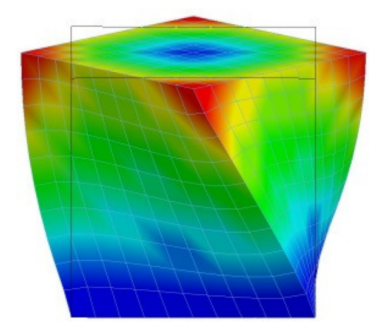

(e) $v=0.499$

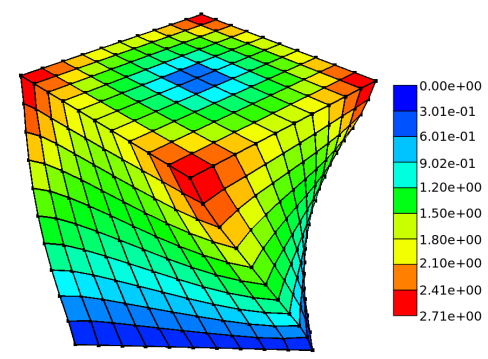

(c)

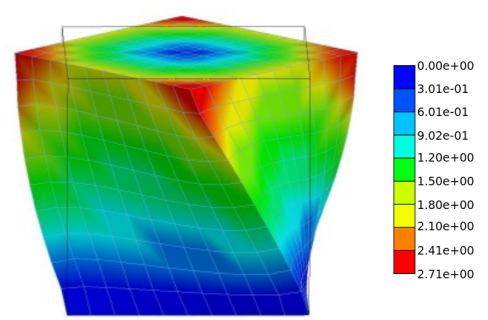

(f)

Fig. 9 Torsion experiment with big (20\%) deformation at different Poisson's ratios, $E=10,000$; comparison between HybMSS and FEM. The color scale corresponds to the displacement from the original position.

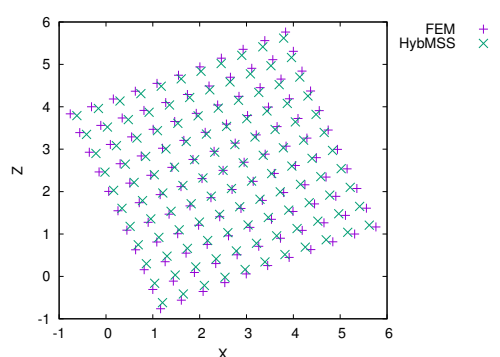

(a) $v=0$

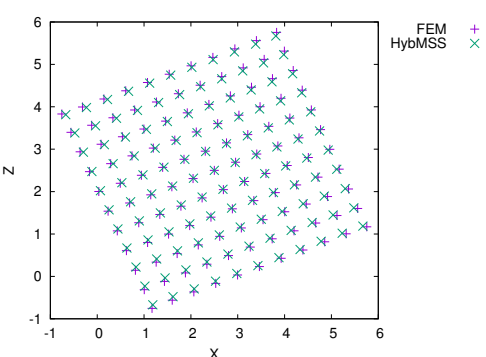

(b) $v=0.25$

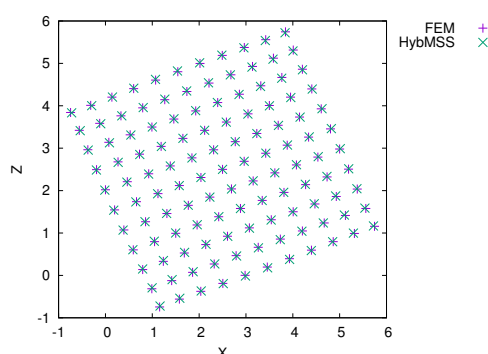

(c) $v=0.499$

Fig. 10 Shape of the body for the torsion experiment with big (20\%) deformation at different Poisson's ratios, $E=10,000$; comparison between HybMSS and FEM.

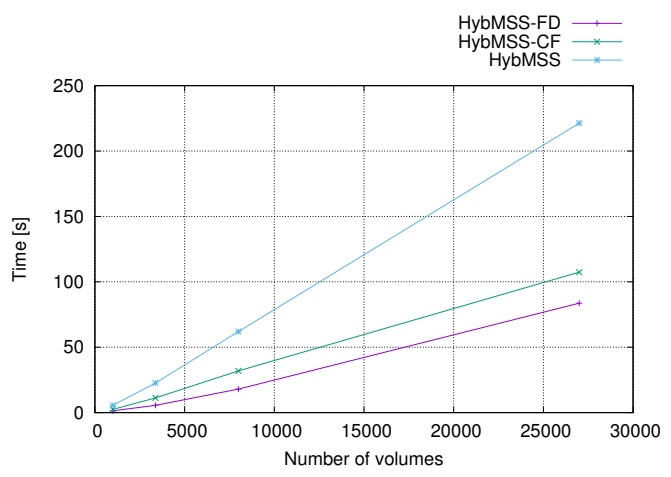

Fig. 11 Real time performance of 1,000 iterations versus the number of mesh elements (volumes) with CPU-software configuration I for the compression experiment and the simulation methods: HybMSS-FD, HybMSS-CF, HybMSS.

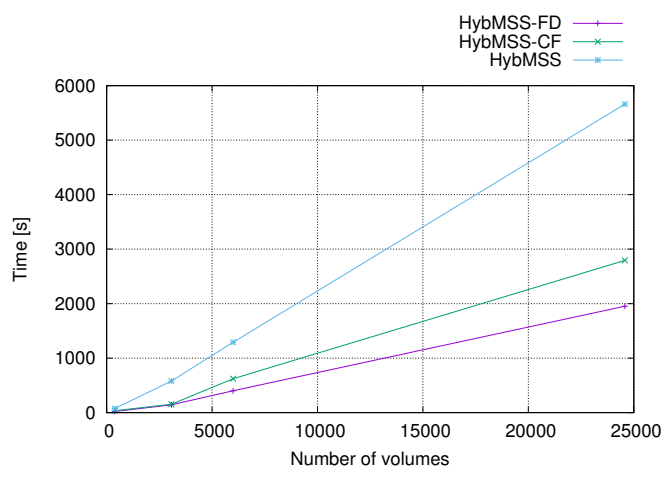

Fig. 12 Real time execution of 300,000 iterations versus the number of mesh elements (volumes) on the CPU-software configuration I for the beam under gravity experiment and the three simulation methods: HybMSS-FD, HybMSS-CF, HybMSS. 


\begin{tabular}{|c|c|c|c|c|}
\hline \multirow{2}{*}{ Mesh size } & \multirow{2}{*}{ \#volumes } & \multirow{2}{*}{ \#vertices } & \multicolumn{2}{|c|}{ \#springs } \\
\cline { 4 - 5 } & & & no FD & with FD \\
\hline $10 \times 10 \times 10$ & 1,000 & 1,331 & 7,630 & 19,630 \\
$15 \times 15 \times 15$ & 3,375 & 4,096 & 25,020 & 65,520 \\
$20 \times 20 \times 20$ & 8,000 & 9,261 & 58,460 & 154,460 \\
$30 \times 30 \times 30$ & 27,000 & 29,791 & 194,490 & 518,490 \\
\hline \hline $4 \times 4 \times 24$ & 384 & 625 & 3,136 & 7,744 \\
$8 \times 8 \times 48$ & 3,072 & 3,969 & 23,232 & 60,096 \\
$10 \times 10 \times 60$ & 6,000 & 7,381 & 44,680 & 116,680 \\
$16 \times 16 \times 96$ & 24,576 & 28,033 & 178,816 & 473,728 \\
\hline
\end{tabular}

Table 6 Details of the meshes used in the performance experiments. no FD is the number of springs without face diagonal springs, while with FD is the number of springs including the face diagonal springs.

In average, HybMSS-CF is 1.4 times slower than HybMSSFD, due to the additional springs. HybMSS is 2.1 times slower than HybMSS-FD, due to the additional computation done in order to compute the correction forces. Note that this computation is not yet parallelized.

\subsection{To Summarize}

To sum up, we saw that the use of correction forces and face diagonal springs in mass-spring systems plays a huge role in the body behavior and the shape of resulting deformation. The presented experimental results show that HybMSS creates natural deformations of shapes expected by the physical laws unlike the HybMSS-FD system, which lacks realistic shape deformation provided by the face-diagonal springs. Additionally the numerical results indicate that it is possible to create a mass-spring model which ensures the correct compressibility rate in a sample locally throughout all volumes and not just as an average result.

\section{Conclusions and perspectives}

In this paper we introduced improvements to the classical MSS used to simulate isotropic tissues. The proposed contributions include avoiding the wrinkle instability and introducing the physically correct stiffness formulations for all the used types of springs and extension of the MSS to use all the real values of Poisson's ratio. Working on cubicalsymmetrical meshes, we shown the effect of face-diagonal springs on the simulation; presented and validated the new stiffness computation, which incorporates the mechanical information about a body into the model; and we shown how to locally correct volumes in order to keep the simulation physically correct with adequate Poisson's ratio.

The model presented in this article is straight-forward in the usage, it has adjustable parameters and succeeds to simulate incompressible isotropic materials (presented $v=$ 0.499) with a minimal error. The basic formulation of the force can be modified according to the type of material and type of simulation that the user wants to perform (like linear or non-linear body behavior). Additionally, the full range of Poisson's ratio values can be used while simulating isotropic bodies thanks to the formulation, which escapes Cauchy's limitations. There are no additional structures introduced into MSS other than its classical springs and the user is finally able to model the behavior of isotropic bodies with various Young's modulus and Poisson's ratio values.

The solution is suitable for real-time applications (the mean performance of 10 second simulation of HybMSS on a sample of 1,000 elements on Intel ${ }^{\circledR}$ Core $^{\mathrm{TM}_{\mathrm{i}}}$ - 4790K CPU@ $4.00 \mathrm{GHz}$ processor is $2.44 \mathrm{~s}$ for $v=1 / 4$ and $5.579 \mathrm{~s}$ for $v \neq 1 / 4$ ), however the additional computation of the volume and correction forces on every element obviously worsens the performance. It is possible to improve the computation time using TBB or consider other parallelization.

We are still working on additional features, especially adjusting the correction forces to work with different base shapes in 3D (tetrahedra, prisms, pyramids). We also aim to accurately simulate behavior of real soft tissues, like liver, spleen and kidney, which will be much more successful using incompressible simulation model combined with a nonlinear force formulation.

\section{APPENDIX A. Theoretical notions of linear elasticity}

\section{A.1. The multi-constant theory of elasticity}

Consider a deformation that brings a material point from its rest position $\mathbf{x} \in \mathbb{R}^{3}$ to a new position $\mathbf{x}+\mathbf{u}(\mathbf{x})$. Obviously, a homogeneous displacement $\mathbf{u}(\mathbf{x})=$ cst amounts to a uniform translation, generating no stress in the material, so the lowest-order quantity of interest in elasticity is the displacement gradient $\boldsymbol{\nabla u}$, the symmetric part of which is called the strain tensor defined by:

$\boldsymbol{\varepsilon}=\frac{1}{2}\left(\boldsymbol{\nabla} \mathbf{u}+(\boldsymbol{\nabla} \mathbf{u})^{\mathrm{T}}\right), \quad \varepsilon_{i j}=\varepsilon_{j i}=\frac{1}{2}\left(\frac{\partial u_{i}}{\partial x_{j}}+\frac{\partial u_{j}}{\partial x_{i}}\right)$.

In linear elasticity, the deformation energy is a quadratic function of the deformation tensor, its volume density being written

$W=\frac{1}{2} C_{i j k l} \varepsilon_{i j} \varepsilon_{k l}$,

where the fourth-rank tensor $\mathbf{C}$ is the stiffness tensor. [We use the implicit summation convention of repeated Cartesian indices $i, j, k$ and $l$ over the values 1, 2 and 3.] The antisymmetric part of $\nabla \mathbf{u}$, which corresponds to solid-body rotations, does not enter expression (7.2) since the deformation energy is invariant with respect to such rotations. Moreover, since $\varepsilon_{i j}=\varepsilon_{j i}$, one has

$C_{i j k l}=\frac{\partial^{2} W}{\partial \varepsilon_{i j} \partial \varepsilon_{k l}}=C_{j i k l}=C_{i j l k}=C_{k l i j}$. 
The stress tensor $\boldsymbol{\sigma}$ derives from the energy:

$\sigma_{i j}=\frac{\partial W}{\partial \varepsilon_{i j}}=\mathrm{C}_{i j k l} \varepsilon_{k l}$

the component $\sigma_{i j}$ being the elastic force along the $i$-direction transmitted across a unit area of a surface normal to the $j$ direction. This proportionality relation between stress and strain is Hooke's law.

\section{A.2. Relations for an isotropic material}

Among the $3^{4}=81$ components of the stiffness tensor $\mathbf{C}$, only 21 are independent in the general case due to relations (7.3) [23]. Symmetries of the material further reduce this number; the most symmetric materials being isotropic, in which case $C^{(i s o)}$ is independent of the orientation of the reference axes and can be written

$\mathrm{C}_{i j k l}^{(i s o)}=\lambda \delta_{i j} \delta_{k l}+G\left(\delta_{i k} \delta_{j l}+\delta_{i l} \delta_{j k}\right)$

in terms of the two Lamé constants $\lambda$ and $G$, where $G$ is named the shear modulus (the relation between stiffness tensor and material symmetry is derived in [23].) The deformation energy of an isotropic material then reads

$W^{(i s o)}=\frac{\lambda}{2} \operatorname{Tr}(\varepsilon)^{2}+G \operatorname{Tr}(\varepsilon)^{2}=\frac{\lambda}{2}\left(\varepsilon_{k k}\right)^{2}+G \varepsilon_{i j} \varepsilon_{i j}$

and the stress tensor is

$\sigma_{i j}^{(i s o)}=\lambda \delta_{i j} \varepsilon_{k k}+2 G \varepsilon_{i j}$

Besides, an alternative set of elastic constants, widely used to wholly describe an isotropic elastic material, is the Young modulus $E$ and the Poisson ratio $v$ which are related to Lamé parameters by:

$E=G \frac{3 \lambda+2 G}{\lambda+G} \quad$ and $\quad v=\frac{\lambda}{2(\lambda+G)}$.

In particular, they are most useful in uniaxial stress situations: in a stress state such that the only non-zero stress component is $\sigma_{x x}^{(i s o)}$, the non-zero strain components are $\varepsilon_{x x}=$ $\sigma_{x x}^{(i s o)} / E$ and $\varepsilon_{z z}=\varepsilon_{y y}=-v \varepsilon_{x x}$.

A last elastic constant of interest is the bulk modulus

$K=\lambda+\frac{2 G}{3}$

such that, in an uniform dilation $\varepsilon_{i j}=\frac{1}{3} \delta_{i j} \Delta V / V$ changing volume $V$ by $\Delta V=V \varepsilon_{k k}$, the stress is $\sigma_{i j}^{(i s o)}=K \Delta V / V \delta_{i j}=$ $K \varepsilon_{k k} \delta_{i j}$.

Acknowledgements This work was supported by the LABEX PRIMES (ANR-11-LABX-0063) of Université de Lyon, within the program "Investissements d'Avenir" (ANR-11-IDEX-0007) operated by the French National Research Agency (ANR). The authors would like to thank for support of E. Flechon for providing the original version of TopoSim (software implementing the LCC+MSS model).

\section{References}

1. Arnab, S., Raja, V.: Chapter 4: Simulating a deformable object using a surface mass spring system. In: 2008 3rd International Conference on Geometric Modeling and Imaging, pp. 21-26 (2008). DOI 10.1109/GMAI.2008.24

2. Baraff, D., Witkin, A.: Large steps in cloth simulation. In: Proceedings of the 25th Annual Conference on Computer Graphics and Interactive Techniques, SIGGRAPH '98, pp. 43-54. ACM, New York, NY, USA (1998). DOI 10.1145/280814.280821. URL http://doi.acm.org/10.1145/280814.280821

3. Baudet, V., Beuve, M., Jaillet, F., Shariat, B., Zara, F.: Integrating Tensile Parameters in Hexahedral Mass-Spring System for Simulation. In: WSCG'2009 (2009)

4. Bender, J., Müller, M., Otaduy M. A., Teschner, M. : Positionbased Methods for the Simulation of Solid Objects in Computer Graphics. In STAR Proceedings of Eurographics, 2013.

5. Born, M., Huang, K.: The Dynamical Theory of Crystal Lattices. Oxford University Press, London (1954)

6. Bourguignon, D., Cani, M.P.: Controlling Anisotropy in MassSpring Systems, pp. 113-123. Springer Vienna, Vienna (2000)

7. Bridson, R., Fedkiw, R., Anderson, J.: Robust treatment of collisions, contact and friction for cloth animation. ACM Trans. Graph. 21(3), 594-603 (2002)

8. Cauchy, A.L.: De la pression ou tension dans un système de points matériels, CEuvres complètes, vol. série 2, tome 8, 1882-1974.

9. Cauchy, A.L.: Sur l'équilibre et le mouvement d'un système de points matériels sollicités par des forces d'attraction ou de répuslion mutuelle, Exercices de Mathématiques, vol. 3, 18821974.

10. Chen, Y., Zhu, Q., Kaufman, A.: Physically-based animation of volumetric objects. In: In Proceedings of IEEE Computer Animation 98, pp. 154-160 (1998)

11. Diziol, R., Bender, J., Bayer, D.: Robust real-time deformation of incompressible surface meshes. In: Proceedings of the 2011 ACM SIGGRAPH/Eurographics Symposium on Computer Animation, SCA '11, pp. 237-246. ACM, New York, NY, USA (2011)

12. Duan, Y., Huang, W., Chang, H., Chen, W., Zhou, J., Teo, S.K., Su, Y., Chui, C., Chang, S.: Volume Preserved Mass-spring Model with Novel Constraints for Soft Tissue Deformation. IEEE journal of biomedical and health informatics 2194(c), 1-12 (2014). DOI 10.1109/JBHI.2014.2370059

13. Elcoro, L., Etxebarria, J.: Common misconceptions about the dynamical theory of crystal lattices: Cauchy relations, lattice potentials and infinite crystals. Eur. J. Phys. 32, 25-35 (2011) DOI 10.1088/0143-0807/32/1/003 URL http://stacks.iop.org/0143$0807 / 32 / \mathrm{i}=1 / \mathrm{a}=003$

14. Gelder, A.V.: Approximate simulation of elastic membranes by triangulated spring meshes. Journal of Graphics Tools 3(2), 2141 (1998). DOI 10.1080/10867651.1998.10487490

15. Hallquist, J.O.: LS-DYNA Theory Manual (2006) URL http://www.lstc.com/pdf/ls-dyna_theory_manual_2006.pdf

16. Jarrousse, O., Fritz, T., Dössel, O.: Implicit Time Integration in a Volumetric Mass-Spring System for Modeling Myocardial Elastomechanics, pp. 876-879. Springer Berlin Heidelberg, Berlin, Heidelberg (2010)

17. Keating, P.N.: Effect of Invariance Requirements on the Elastic Strain Energy of Crystals with Applications to the Diamond Structure. Phys. Rev. 145, 637645 (1966). DOI 10.1103/PhysRev.145.637. URL https://link.aps.org/doi/10.1103/PhysRev.145.637

18. Keating, P.N.: Relationship between the macroscopic and microscopic theory of crystal elasticity. I. Primitive crystals. Phys. Rev. 152, 774-779 (1966). DOI 10.1103/PhysRev.152.774. URL https://link.aps.org/doi/10.1103/PhysRev.152.774

19. Kirkwood, J.G.: The Skeletal Modes of Vibration of Long Chain Molecules. Journal of Chemical Physics 7, 506-509 (1939). DOI $10.1063 / 1.1750479$ 
20. Kot, M., Nagahashi, H.: Second degree of freedom of elastic objects - adjustable Poisson's ratio for mass spring models. In: GRAPP 2015 - Proceedings, Berlin, Germany, 1114 March, 2015, pp. 138-142. SciTePress (2015). DOI http://dx.doi.org/10.5220/0005303601380142

21. Kot, M., Nagahashi, H.: Mass spring models with adjustable Poisson's ratio. Vis. Comput. 33(3), 283291 (2017). DOI 10.1007/s00371-015-1194-8. URL https://doi.org/10.1007/s00371-015-1194-8

22. Kot, M., Nagahashi, H., Szymczak, P.: Elastic moduli of simple mass spring models. Vis. Comput. 31(10), 13391350 (2015). DOI 10.1007/s00371-014-1015-5. URL http://dx.doi.org/10.1007/s00371-014-1015-5

23. Lifshitz, E., Kosevich, A., Pitaevskii, L.: Theory of elasticity (third edition). Butterworth-Heinemann, Oxford (1986)

24. Lloyd, B.A., Székely, G., Harders, M.: Identification of spring parameters for deformable object simulation. IEEE Transactions on Visualization and Computer Graphics 13(1), 1081-1093 (2007). DOI 10.1109/TVCG.2007.1055

25. Marchal, M.: Soft tissue modeling for computer assisted medical interventions. Theses, Université Joseph-Fourier - Grenoble I (2006). URL https://tel.archives-ouvertes.fr/tel-00129430

26. Mollemans, W., Schutyser, F., Van Cleynenbreugel, J., Suetens, P.: Tetrahedral Mass Spring Model for Fast Soft Tissue Deformation, pp. 145-154. Springer Berlin Heidelberg, Berlin, Heidelberg (2003)

27. Natsupakpong, S., Çavuşoğlu, M. Cenk: Determination of elasticity parameters in lumped element (mass-spring) models of deformable objects. Graphical Models 72(6), 61-73 (2010). DOI 10.1016/j.gmod.2010.10.001

28. Niiranen, J.: Fast and accurate symmetric Euler algorithm for electromechanical simulations. IMACS 1, 71-78 (1999)

29. Ostoja-Starzewski, M., Sheng, P.Y., Alzebdeh, K.: Spring network models in elasticity and fracture of composites and polycrystals. Comput. Mater. Sci. 7, 82-93 (1996)

30. Sahimi, M., Arbabi, S.: Mechanics of disordered solids. II. Percolation on elastic networks with bond-bending forces. Physical Review B 47, 703-712 (1993). DOI 10.1103/PhysRevB.47.703. URL https://link.aps.org/doi/10.1103/PhysRevB.47.703

31. de Saint-Venant, A.J.C.B.: De la torsion des prismes, avec des considérations sur leur flexion ainsi que sur l'équililbre des solides élastiques en général et des formules pratiques pour le calcul de leur résistance à divers efforts s'exerçant simultanément. No. 14 in Mémoires présentés par divers savants à l'Académie des Sciences de l'Institut Impérial de France. Académie des Sciences de l'Institut Impérial de France (1855). URL https://gallica.bnf.fr/ark:/12148/bpt6k99739z/f1.image

32. San-Vicente, G., Aguinaga, I., Tomás Celigüeta, J.: Cubical Mass-Spring Model design based on a tensile deformation test and nonlinear material model. IEEE transactions on visualization and computer graphics 18(2), 228-41 (2012). DOI 10.1109/TVCG.2011.32

33. Terzopoulos, D., Platt, J., Fleischer, K.: Heating and melting deformable models. The Journal of Visualization and Computer Animation 2(2), 68-73 (1991)

34. Todhunter, I.: A History of the Theory of Elasticity and of the Strength of Materials: From Galilei to the Present Time, Cambridge Library Collection - Mathematics, vol. 2. Cambridge University Press (2014). DOI 10.1017/CBO9781107280076

35. Vincente-Otamendi, G.S.: Designing deformable models of soft tissue for virtual surgery planning and simulation using the MassSpring Model. Ph.D. thesis, Universidad de Navarra (2011) 